\title{
A 90-Day Repeated Oral Dose Toxicity Study of Alismatis Rhizoma Aqueous Extract in Rats
}

\author{
Mu-Jin Lee ${ }^{1,2}$, Ho-Kyung Jung', Ki-Ho Lee', Ji-Hun Jang', Mi-Ok Sim ', Tea-Gyeong Seong', \\ Byung-Kwan Ahn' ${ }^{1}$, Jin-Han Shon', Seong-Ho Ham', Hyun-Woo Cho', Yong-Min Kim³, \\ Sung-Jin Park ${ }^{3}$, Ji-Young Yoon ${ }^{4}$, Je-Won $\mathrm{Ko}^{2}$ and Jong-Choon Kim ${ }^{2}$ \\ ${ }^{1}$ Division of Tradition Korean Medicine Research, National Development Institute of Korean Medicine, Jangheung, \\ Korea \\ ${ }^{2}$ College of Veterinary Medicine BK21 Plus Project Team, Chonnam National University, Gwangju, Korea \\ ${ }^{3}$ Preclinical Research Center, MEDVILL Co., Seoul, Korea \\ ${ }^{4}$ Jeonju AgroBio-Materials Institute, Jeonju, Korea
}

\begin{abstract}
Alismatis rhizoma (AR), the dried rhizome of Alisma orientale (Sam.) Juzep, is a well-known, traditional medicine that is used for the various biological activities including as a diuretic, to lower cholesterol and as an antiinflammatory agent. The present study was carried out to investigate the potential toxicity of the Alismatis rhizoma aqueous extract (ARAE) following 90-day repeated oral administration to Sprague-Dawley rats. ARAE was administered orally to male and female rats for 90 days at 0 (control), 500, 1,000 and 2,000 mg/kg/day $(n=10$ for male and female rats for each dose). Additional recovery groups from the control group and high dose group were observed for a 28-day recovery period. Chromatograms of ARAE detected main compounds with four peaks. Treatment-related effects including an increase in the red blood cells, hemoglobin, hematocrit, albumin, total protein, and urine volume were observed in males of the $2,000 \mathrm{mg} / \mathrm{kg} /$ day group $(p<0.05)$. However, the diuretic effect of ARAE was considered, a major cause of hematological and serum biochemical changes. The oral noobserved-adverse-effect level (NOAEL) of the ARAE was $>2,000 \mathrm{mg} / \mathrm{kg} / \mathrm{day}$ in both genders, and no target organs were identified.
\end{abstract}

Key words: Alismatis rhizome aqueous extract, Traditional medicine, Diuretic effect, Sub-chronic toxicity, NOAEL

\section{INTRODUCTION}

Traditional herbal medicines are generally regarded as safe because they have long been used for various pur-

Correspondence to: Jong-Choon Kim, College of Veterinary Medicine BK21 Plus Project Team, Chonnam National University, 77 Yongbong-ro, Buk-gu, Gwangju 61186, Korea

E-mail: toxkim@jnu.ac.kr

Hyun-Woo Cho, Division of Tradition Korean Medicine Research, National Development Institute of Korean Medicine, 288 Udeuraendeugil, Anyang-myeon, Jangheung, Jeonnam 59338, Korea E-mail: johw7@nikom.or.kr

This is an Open-Access article distributed under the terms of the Creative Commons Attribution Non-Commercial License (http:// creativecommons.org/licenses/by-nc/3.0) which permits unrestricted non-commercial use, distribution, and reproduction in any medium, provided the original work is properly cited. poses, but this is not always confirmed scientifically (1). Alismatis rhizome (AR, common name water plantain or Taeg-Sa in Korea) is a perennial herb that originates from the rhizomes of Alisma orientale Juzepzuk (Alismataceae) $(2,3)$. Alismatis orientale is a water plant of the Alismataceae family, which contains approximately 90 species and 11 genera worldwide but is distributed primarily across East Asia (4). The plants are reproduced by seedling-raising, which has the characteristic of seeding in summer and harvesting in winter (5). Traditional medicine Alismatis rhizome is the dried rhizome of the fresh plant Alisma. AR has been widely used as an important ingredient in a number of traditional Korean medicine formulations, such as Taeg-Sa-San, O-Ryeing-San and Yuk-Mi-Ji-Hwang-Tang and is believed to lower cholesterol, polydipsia, pedal edema, act as a diuretic, and have anti-allergic, anti-inflammatory, and antibacterial properties (6-9). An inhibitory effect on 
renal stone formation of AR has been demonstrated by some modern pharmacological studies in rats (10).

AR compounds that have been discovered include terpenoids (11), phenolic (12), polysaccharides (13), carbohydrates, proteins, amino acids, etc (5). Diuretic activity investigations have shown that the alisol A content in AR has a similar diuretic effect with hydrochlorothiazide, and alisol A 24-acetate and hydrochlorothiazide increased the excretion of $\mathrm{Na}^{+}$and $\mathrm{K}^{+}$in rats (14).

Recent toxicological studies have been generally indicated the safety of AR (15-19). Huang et al. (17) have reported that oral administration of the lipid-soluble fraction (i.e., triterpene-enriched extract) of AR at levels up to $1,440 \mathrm{mg} / \mathrm{kg} / \mathrm{day}$ for 90 days does not cause adverse effects or mortality in rats. Although the safety of its water-soluble fraction has also been confirmed by some recent studies $(15,16)$, there is no information in the published literature regarding the potential sub-chronic toxicity of the water-soluble fraction, designed in accordance with the current regulatory guidelines. Thus, a systematic study on the safety of AR is essential for increased public use.

Therefore, as part of a project to provide safety data of the $\mathrm{AR}$ required for its use as natural medicine or functional foods and drugs, the present study was conducted according to the test guidelines from the Korea Food and Drug Administration (KFDA) and Organisation for Economic Cooperation and Development (OECD) guidelines for the testing of chemicals under modern Good Laboratory Practice Regulations. AR aqueous extract (ARAE) was orally administered to male and female Sprague-Dawley rats at 0 (control), 500, 1,000 and 2,000 mg/kg/day for 90 consecutive days. Physical changes, estrus cycles, sperm parameters, urinalysis, hematology, serum biochemistry, organ weight, and histopathological changes were measured to determine gross toxicity, the no-observed-adverse-effect level (NOAEL), and target organs.

\section{MATERIALS AND METHODS}

Test material. AR was collected from Suncheon Province (Jeonman, Korea), and was purchased from the Herb medicine farming association (HMFA) and identified by the National Development Institute for Traditional Korean Medicine as A. orientalis (Sam.) Juzep. The AR (5 kg) was extracted twice by decoction with distilled water $(50 \mathrm{~L})$ for $4 \mathrm{hr}$ at $95^{\circ} \mathrm{C}$, and the solution was filtered with a 100 mesh sieve. The ARAE, freeze-dried powder was concentrated in vacuo $\left(5-10 \mathrm{hPa}\right.$ at $\left.50-60^{\circ} \mathrm{C}\right)$ and lyophilized using a freeze-drying system (Lyoph-pride 20R, Ilshin, Yangju, Korea). Total ARAE powder acquired was $1.197 \mathrm{~kg}$ (yield $23.9 \%$ ).

UFLC-IT-TOF MS analysis. The LC analyses were performed on a Shimadzu analytical UFLC system (Shi- madzu, Kyoto, Japan) consisting of two LC-20AD XR pumps (pump A and pump B), a CTO-20A column oven, a DGU-20A $\mathrm{A}_{3}$ degasser, a SPD-20A UV detector, and an SIL-20A XR autoinjector. An ACQUITY UPLC ${ }^{\circledR}$ BEH C18 column (1.7 $\mu \mathrm{m}, 2.1 \mathrm{~mm}, 150 \mathrm{~mm}$, Waters, FL, USA) with a column temperature of $40^{\circ} \mathrm{C}$ was used for sample separation. The UFLC mobile phases were pumped by pump A (water) and pump B (acetonitrile), respectively; the water phase contained $0.1 \%$ formic acid, and the flowrate was $0.3 \mathrm{~mL} / \mathrm{min}$. The on-line chromatograms of the ARAE were recorded by UV1 at $254 \mathrm{~nm}$ in the UFLC system. The above UFLC system was connected to a hybrid IT-TOF mass spectrometer (LCMS-IT-TOF, Shimadzu) via an ESI interface. The detector voltage was fixed at $1.67 \mathrm{kV}$ and the curved desolvation line (CDL) temperature and block heater temperature were both maintained at $200^{\circ} \mathrm{C}$. The flowrate of nitrogen as nebulizer gas was $1.5 \mathrm{~L} /$ min. For full scan MS analyses, spectra were recorded in the range $\mathrm{m} / \mathrm{z} 100-1,000$. Data were processed later by LC/MS solution software (Version 3, Shimadzu), which included a formula predictor.

Animal husbandry and maintenance. Fifty-five Sprague-Dawley rats of each sex were obtained from the DBL Inc. (Eumseong, Chungbuk, Korea) at 5 weeks of age, and used following 2 weeks of quarantine and acclimatization. The animals were housed in a room maintained at a temperature of $19-25^{\circ} \mathrm{C}$ and a relative humidity of 30 $70 \%$, with artificial lighting from 07:00 to 19:00 (150300 Lux) and 10-15 air changes per hr. Only healthy animals were included in the study. The animals were kept in solid-bottomed polycarbonate cages and allowed sterilized tap water and commercial rodent chow (Zeigler ${ }^{\circledR}$, PA, USA) ad libitum. The animals were maintained in accordance with the Guide for the care and Use of Laboratory Animals, and also approved by the animal experiment committee of National Development Institute of Korean Medicine on the basis of the Animal Protection Act.

Selection of doses and treatment. A dose of 2,000 $\mathrm{mg} / \mathrm{kg} /$ day was set as the high dose based on the results of a preliminary 2-week repeated oral dose toxicity study (19) and the characteristics of the natural test substance. Doses of 1,000 and $500 \mathrm{mg} / \mathrm{kg} /$ day were set as the middle and low doses, respectively, using a common ratio of 2 . The vehicle control group was treated with sterile distilled water for injection only. Each group consisted of 10 rats per gender. The test substance was administered repeatedly by oral route to each group for 90 days. Dosing volume was set to $10 \mathrm{~mL} / \mathrm{kg}$ for the repeated dose toxicity study, based on the most recent body weights.

Animal observation. All animals were observed twice daily for mortality, general condition and clinical signs 
before the test and throughout dosing period. All animals were weighed individually just prior to dosing on day 1 and once a week thereafter. Body weight data from the day of necropsy was not included in the evaluation of body weights, because these data reflected the fasted body weight of the animals. Daily food consumption was measured before the initiation of dosing and once a week thereafter. Food consumption was calculated by subtracting leftover feed from the total feed provided.

Urinalysis. Five rats per group were housed in metabolic cages for urine collection in the last week of observation, and fresh urine samples were collected for $3 \mathrm{hr}$ and used for urinalysis including urine volume. Test strips (Multistix 10SG, Siemens, Washington, DC, USA) were dipped in about $0.3 \mathrm{~mL}$ of urine and glucose, bilirubin, ketone bodies, specific gravity, occult blood, $\mathrm{pH}$, protein, urobilinogen, and nitrite were analyzed with an automatic analyzer (Clini-Tek 100, Siemens, Ames Division, Miles Laboratory, NY, USA). Electrolytes including sodium $\left(\mathrm{Na}^{+}\right)$, potassium $\left(\mathrm{K}^{+}\right)$, and chloride $\left(\mathrm{Cl}^{-}\right)$were measured with an electrolyte auto-analyzer (FUJI DRI-CHEM 4000i, FUJI, Tokyo, Japan).

Hematology and serum biochemistry. All animals were fasted for at least $18 \mathrm{hr}$ before necropsy, when blood samples were collected from the abdominal aorta. Whole blood was placed into a CBC bottle containing EDTA-2K anticoagulant. The following parameters were analyzed with a Coulter counter (XN 9000, Sysmex America, Inc., IL, USA): erythrocyte count (RBC), hemoglobin (HGB), hematocrit (HCT), mean corpuscular volume (MCV), mean corpuscular hemoglobin (MCH), mean corpuscular hemoglobin concentration (MCHC), platelet count (PLT), total leucocyte counts (WBC), and WBC differential count [neutrophils (NEU, \%), lymphocytes (Lym, \%), monocytes (MONO, \%), eosinophils (EOS, \%) and basophils (Baso, $\%)$ ]. Blood for serum biochemistry was collected into a Vacutainer tube containing a clot activator. The blood was coagulated by maintaining at room temperature for 30-40 min followed by centrifugation for $10 \mathrm{~min}$. The following parameters were measured with a serum biochemistry analyzer (Hitachi 7020, HITACHI, Tokyo, Japan): aspartate aminotransferase (AST), alanine aminotransferase (ALT), alkaline phosphatase (ALP), creatine phosphokinase (CPK), gamma glutamyltranspeptidase (GGT), blood urea nitrogen (BUN), glucose (GLU), albumin (ALB), total protein (TP), A/G (albumin/globulin) ratio, total cholesterol (T-CHO), creatinine (CRE), triglycerides (TG), inorganic phosphorus (IP) and calcium ions (Ca).

Vaginal cytology. Five female rats in each group were evaluated on weeks 11 and 12 of the study. Vaginal smears were collected every morning for 14 days, stained with Diff-Quick solution, and observed microscopically. The estrus cycle reflected by the vaginal smear was characterized as proestrus, estrus, metestrus or diestrus according to the features of each cell type.

Sperm evaluation. Five pre-selected males per group were evaluated at necropsy. The right testis was placed in $10 \mathrm{~mL}$ of distilled water, homogenized for one minute and sonicated for three minutes (Kinematica, LU, Swiss). One drop of the homogenization-resistant sperm heads solution was placed onto the sperm number Makler counting chamber (Sefi Medical Instrument, HFA, Israel) and total sperm counts for each animal were determined using a microscope. The number of sperm per $1 \mathrm{~g}$ of right testis was calculated. The left caudal epididymis was incised, weighed and placed in Dulbecco's Phosphate Buffered Saline containing $1 \%$ bovine serum albumin. Sperm was incubated for approximately 5 to $10 \mathrm{~min}$ in culture media. Sperm samples were smeared on glass slides, stained with DiffQuick and examined for morphology using a light microscope, a $200 \mathrm{sperm} / \mathrm{smear}$. Malformations were calculated as follows: Sperm malformation $(\%)=($ Number of abnormal sperm/number of total sperm $) \times 100$.

Necropsy, organ weight and histopathology. Gross post-mortem examinations of the organs in the cranial, thoracic, and abdominal cavities of the sacrificed rats were performed. Absolute and relative weights (organ to body weight ratio) of organs were measured; these included the liver, heart, spleen, lung, kidneys, adrenal glands, ovaries, uterus, testes and epididymides. Histopathological examinations were performed on 5 males and 5 females per group for the main group and recovery group. All gross lesions as defined by the study pathologist were also included in the examination. The testes and epididymides were preserved in Bouin's fixative. The following organs and tissues were fixed in $10 \%$ neutral buffered formalin solution: liver, heart, spleen, lung, kidneys, ovaries and uterus. All preserved organs or tissues were trimmed, processed, embedded in paraffin, sectioned, and stained with hematoxylin \& eosin (H\&E). Specimens were processed for histopathology at the Preclinical Research Center, MEDVILL Co., Ltd. (Seoul, Korea), which is a GLP institute approved by the KFDA. Microscopic examinations were performed in our research center using the Pristi$\mathrm{ma}^{\mathrm{TM}}$ and Path/Tox System (Version 6.3.0, Xybion Medical Systems Co., Cedar Knolls, NJ, USA).

Statistical analysis. Data were presented as means and standard deviation for each group. Body weight, food consumption, hematology, serum biochemistry, estrus cycle, sperm parameters, and organ weight were performed using the SPSS version 12.0 (SPSS, Chicago, IL, USA) with one-way analysis of variance. The homogeneity of variance was examined by Bartlett's test. When variance 
between the groups was confirmed as homogenous, the difference between the control group and each treated group was examined by Duncan's multiple range test.
When variance between the groups was not homogenous, the normal distribution of the date was determined by Kolmogorov-Smirnov test. In case of non-normal distribu-
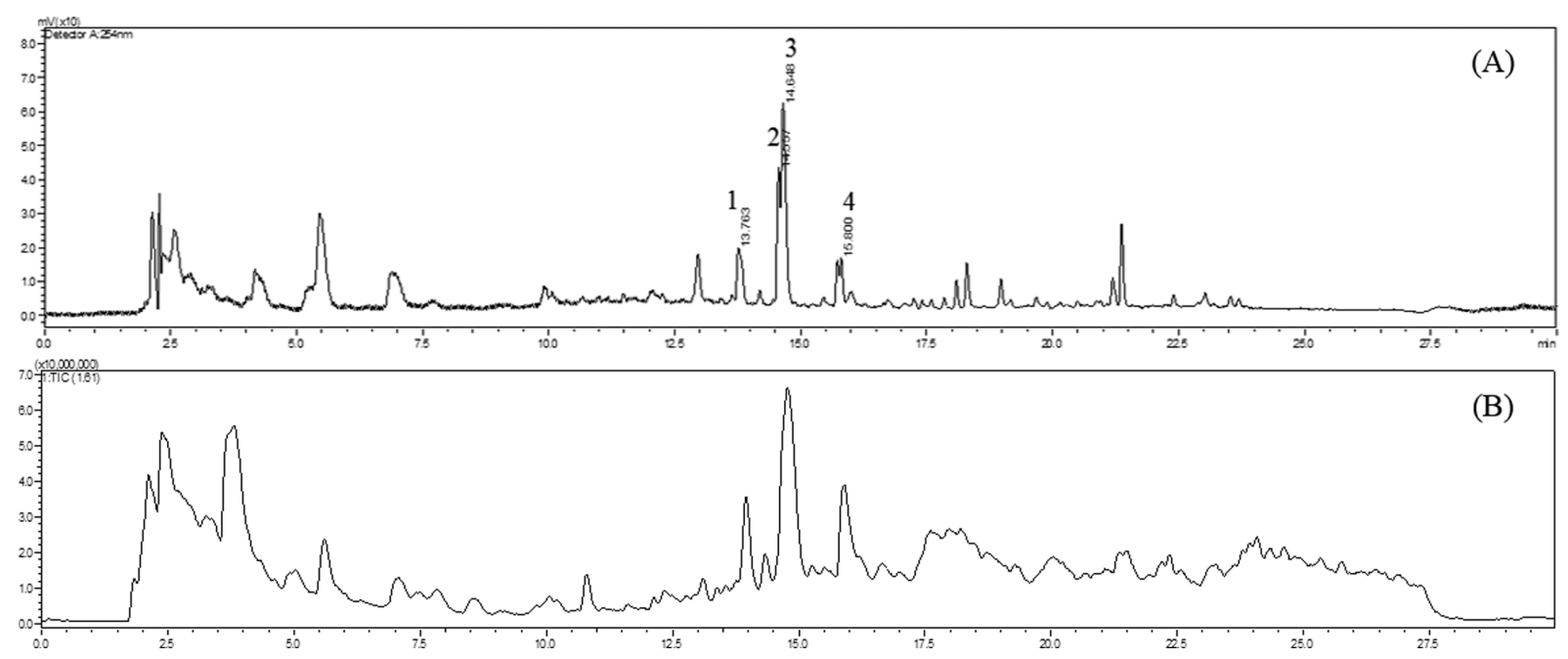

Fig. 1. LC and MS chromatograms of ARAE. (A) UV detector at $254 \mathrm{~nm}$; (B) Total ion chromatograms (TICS).

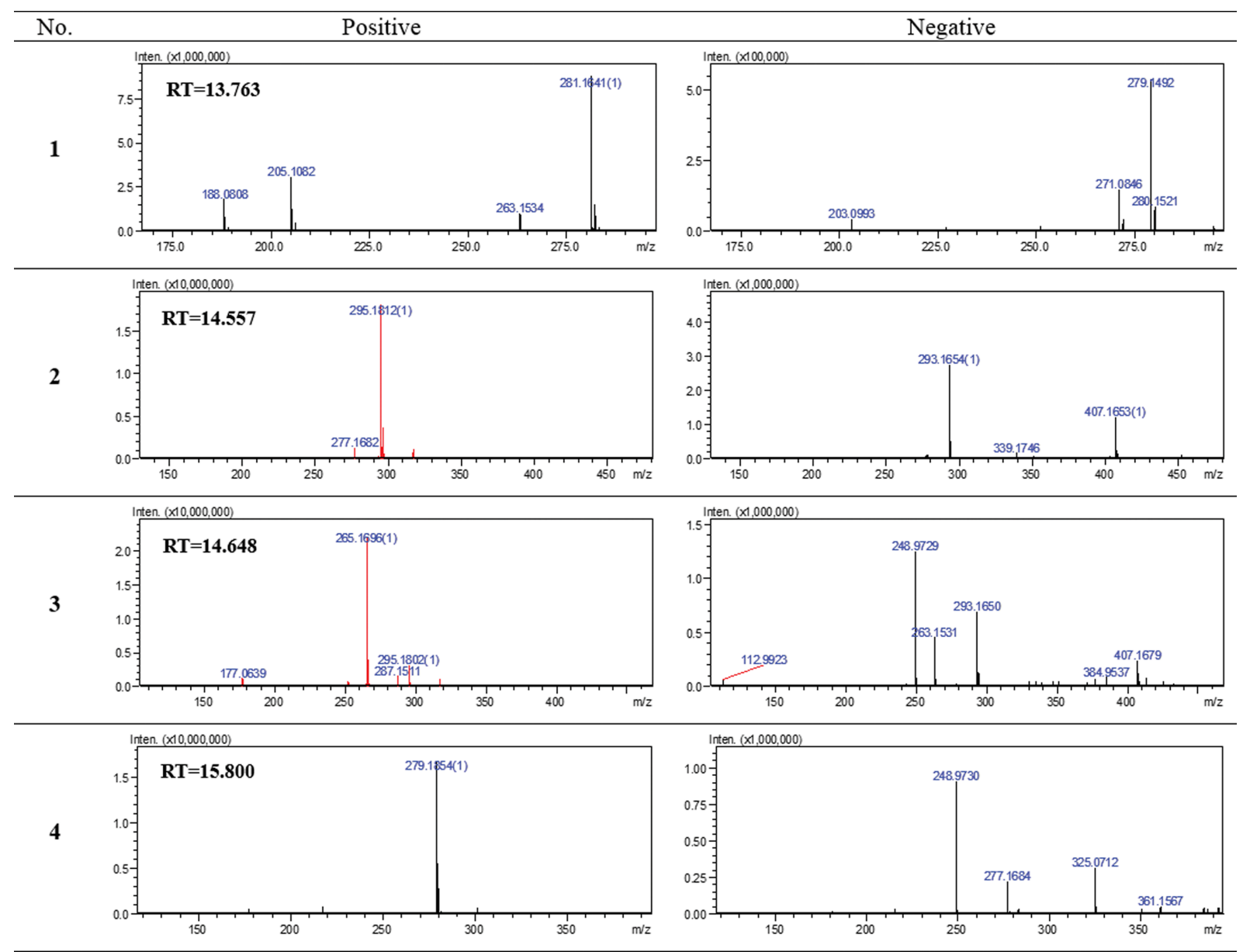

Fig. 2. MS spectrum of chromatogram peaks from ARAE. 
Table 1. Urinalysis findings of male and female rats in the 90-day repeated oral dose toxicity study of ARAE

\begin{tabular}{|c|c|c|c|c|c|c|c|c|}
\hline \multirow{3}{*}{ Parameter } & \multicolumn{8}{|c|}{ Dose (mg/kg/day) } \\
\hline & \multicolumn{4}{|c|}{ Male } & \multicolumn{4}{|c|}{ Female } \\
\hline & 0 & 500 & 1,000 & 2,000 & 0 & 500 & 1,000 & 2,000 \\
\hline \multicolumn{9}{|l|}{ Glucose (mg/dL) } \\
\hline Negative & 4 & 5 & 5 & 5 & 5 & 5 & 5 & 5 \\
\hline 100 (trace) & 1 & 0 & 0 & 0 & 0 & 0 & 0 & 0 \\
\hline \multicolumn{9}{|l|}{ Bilirubin } \\
\hline Negative & 3 & 4 & 3 & 4 & 3 & 5 & 5 & 2 \\
\hline $1+$ & 2 & 1 & 2 & 1 & 2 & 0 & 0 & 2 \\
\hline $2+$ & 0 & 0 & 0 & 0 & 0 & 0 & 0 & 1 \\
\hline \multicolumn{9}{|l|}{ Ketone body (mg/dL) } \\
\hline Negative & 0 & 0 & 1 & 1 & 3 & 5 & 5 & 4 \\
\hline $5($ trace $)$ & 0 & 0 & 0 & 2 & 2 & 0 & 0 & 1 \\
\hline 15 & 4 & 2 & 2 & 1 & 0 & 0 & 0 & 0 \\
\hline 40 & 0 & 3 & 2 & 1 & 0 & 0 & 0 & 0 \\
\hline$\geq 80$ & 1 & 0 & 0 & 0 & 0 & 0 & 0 & 0 \\
\hline \multicolumn{9}{|l|}{ Specific gravity } \\
\hline 1.010 & 0 & 0 & 1 & 0 & 0 & 0 & 0 & 0 \\
\hline 1.015 & 1 & 0 & 0 & 2 & 0 & 2 & 0 & 0 \\
\hline 1.020 & 0 & 1 & 0 & 1 & 0 & 0 & 0 & 0 \\
\hline 1.025 & 0 & 1 & 0 & 1 & 1 & 0 & 1 & 0 \\
\hline$\geq 1.030$ & 4 & 3 & 4 & 1 & 4 & 3 & 4 & 5 \\
\hline \multicolumn{9}{|l|}{ Occult blood } \\
\hline Negative & 2 & 2 & 0 & 2 & 5 & 5 & 4 & 5 \\
\hline Trace intact & 2 & 1 & 1 & 0 & 0 & 0 & 0 & 0 \\
\hline Trace lysed & 0 & 0 & 2 & 3 & 0 & 0 & 1 & 0 \\
\hline $1+$ & 1 & 1 & 2 & 0 & 0 & 0 & 0 & 0 \\
\hline $2+$ & 0 & 0 & 0 & 0 & 0 & 0 & 0 & 0 \\
\hline $3+$ & 0 & 1 & 0 & 0 & 0 & 0 & 0 & 0 \\
\hline \multicolumn{9}{|l|}{$\mathrm{pH}$} \\
\hline 6.0 & 0 & 0 & 0 & 0 & 2 & 1 & 1 & 3 \\
\hline 6.5 & 2 & 2 & 0 & 1 & 2 & 2 & 3 & 2 \\
\hline 7.0 & 2 & 1 & 4 & 2 & 1 & 0 & 1 & 0 \\
\hline 7.5 & 0 & 2 & 1 & 1 & 0 & 2 & 0 & 0 \\
\hline 8.0 & 0 & 0 & 0 & 0 & 0 & 0 & 0 & 0 \\
\hline 8.5 & 1 & 0 & 0 & 1 & 0 & 0 & 0 & 0 \\
\hline \multicolumn{9}{|l|}{ Protein (mg/dL) } \\
\hline Negative & 0 & 0 & 0 & 0 & 0 & 1 & 1 & 0 \\
\hline Trace & 0 & 1 & 1 & 0 & 2 & 1 & 4 & 0 \\
\hline $1+$ & 1 & 0 & 0 & 4 & 2 & 3 & 0 & 3 \\
\hline $2+$ & 3 & 4 & 3 & 1 & 1 & 0 & 0 & 2 \\
\hline $3+$ & 1 & 0 & 1 & 0 & 0 & 0 & 0 & 0 \\
\hline \multicolumn{9}{|c|}{ Urobilinogen (Ehrlich unit/dL) } \\
\hline 0.2 & 5 & 5 & 5 & 5 & 5 & 5 & 5 & 4 \\
\hline 1.0 & 0 & 0 & 0 & 0 & 0 & 0 & 0 & 1 \\
\hline \multicolumn{9}{|l|}{ Nitrite } \\
\hline Negative & 5 & 5 & 5 & 5 & 5 & 5 & 5 & 5 \\
\hline $\mathrm{Na}(\mathrm{mEq} / \mathrm{L})^{\mathrm{a}}$ & $294 \pm 14$ & $286 \pm 18$ & $289 \pm 10$ & $298 \pm 5$ & $293 \pm 7$ & $298 \pm 4$ & $299 \pm 1$ & $294 \pm 8$ \\
\hline $\mathrm{K}(\mathrm{mEq} / \mathrm{L})^{\mathrm{a}}$ & $1.6 \pm 1.4$ & $1.5 \pm 0.7$ & $2.2 \pm 2.6$ & $2.1 \pm 1.6$ & $1.6 \pm 1.4$ & $1.9 \pm 1.5$ & $1.3 \pm 0.6$ & $1.1 \pm 0.1$ \\
\hline $\mathrm{Cl}(\mathrm{mEq} / \mathrm{L})^{\mathrm{a}}$ & $187 \pm 29$ & $185 \pm 21$ & $181 \pm 23$ & $201 \pm 16$ & $185 \pm 25$ & $203 \pm 17$ & $201 \pm 14$ & $181 \pm 10$ \\
\hline Volume $(\mathrm{mL} / 3 \mathrm{hr})^{\mathrm{a}}$ & $0.39 \pm 0.2$ & $0.41 \pm 0.4$ & $0.75 \pm 0.2$ & $1.08 \pm 0.3^{*}$ & $0.24 \pm 0.1$ & $0.26 \pm 0.3$ & $0.39 \pm 0.3$ & $0.54 \pm 0.3$ \\
\hline
\end{tabular}

$\mathrm{Na}$, sodium; $\mathrm{K}$, potassium; and $\mathrm{Cl}$, chloride.

${ }^{a}$ Data are given as mean \pm standard deviation.

*Significantly different from vehicle control at $p<0.05$. 
tion, variance between the groups was examined by Kruskal-Wallis comparison test. When the data were found to be normally distributed, the difference between the control group and each treated group was examined by MannWhitney U-test. $P$ values $<0.05$ were considered significant.

\section{RESULTS}

LC and MS chromatograms of ARAE. Subsequent UFLC-IT-TOF/MS analysis of the ARAE was performed in the positive and negative ion mode. The UV detector and total ion chromatograms (TICs) of ARAE were shown in Fig. 1. Chromatograms of ARAE detected main compounds with eluents at $254 \mathrm{~nm}$. Molecular weights of unknown compounds (4 peaks) were measured by MS spectrum: compound peak 1 (RT $13.763 \mathrm{~min}, \mathrm{~m} / \mathrm{z} 281.1641$ $\left.[\mathrm{M}+\mathrm{H}]^{+}, 279.1492[\mathrm{M}-\mathrm{H}]^{-}\right)$, compound peak 2 (RT 14.557 min, $\left.m / z 295.1812[\mathrm{M}+\mathrm{H}]^{+}, 293.1654[\mathrm{M}-\mathrm{H}]^{-}\right)$, compound peak 3 (RT $14.648 \mathrm{~min}, \mathrm{~m} / z$ 265.1696 [M+H] ${ }^{+}, 263.1531$ $[\mathrm{M}-\mathrm{H}]^{-}$), and compound peak 4 (RT $15.800 \mathrm{~min}, \mathrm{~m} / \mathrm{z}$ $\left.279.1854[\mathrm{M}+\mathrm{H}]^{+}, 277.1684[\mathrm{M}-\mathrm{H}]^{-}\right)$(Fig. 2).

Clinical signs, body weight, and food consumption. No overt signs of toxicity were observed in any animals treated with the test substance. No mortality was observed during the treatment or recovery period. In addition, no significant changes were observed in body weight and food consumption during the treatment or recovery period.

Urinalysis. Urine volume in males of the $2,000 \mathrm{mg} /$ $\mathrm{kg}$ /day group increased significantly when compared with that in the vehicle control group $(p<0.05)$. No significant difference was observed in the qualitative analysis of urine parameters, including glucose, bilirubin, ketone bodies, specific gravity, occult blood, $\mathrm{pH}$, protein, urobilinogen, nitrite, sodium, potassium, and chloride in the urine in both genders of rats collected at the end of treatment (Table 1).

Hematology and serum biochemistry. RBC, HGB, $\mathrm{HCT}$, and MONO in males of the $2,000 \mathrm{mg} / \mathrm{kg} /$ day group increased significantly when compared with those in the vehicle control group $(p<0.05)$. No significant differences in females were observed between the groups. No significant differences were observed in any of the hematological parameters between the groups in either gender at the end of recovery period (Table 2 ).

ALB and TP in males of the $2,000 \mathrm{mg} / \mathrm{kg} /$ day group increased significantly when compared with those in the vehicle control group $(p<0.05)$. No significant differences in females were observed between the groups. No significant differences were observed in any of the serum biochemical values between the groups in either gender at the end of recovery period (Table 3 ).

Vaginal cytology. No significant differences were observed in the length and regularity of estrus cycle between the groups.

Sperm evaluation. In the sperm evaluation of the 0 (control), 500, 1,000 and 2,000 mg/kg/day groups in males, the testicular spermatid head counts were 133.5, 128.9,

Table 2. Hematological values of male and female rats in the 90-day repeated oral dose toxicity study of ARAE

\begin{tabular}{|c|c|c|c|c|c|c|c|c|}
\hline \multirow{3}{*}{ Parameter } & \multicolumn{8}{|c|}{ Dose (mg/kg/day) } \\
\hline & \multicolumn{4}{|c|}{ Male } & \multicolumn{4}{|c|}{ Female } \\
\hline & 0 & 500 & 1,000 & 2,000 & 0 & 500 & 1,000 & 2,000 \\
\hline $\mathrm{RBC}\left(10^{6} / \mu \mathrm{L}\right)$ & $8.87 \pm 0.19$ & $9.38 \pm 0.23$ & $9.13 \pm 0.44$ & $9.53 \pm 0.37 *$ & $8.93 \pm 0.29$ & $8.91 \pm 0.30$ & $8.59 \pm 0.34$ & $8.67 \pm 0.17$ \\
\hline HGB (g/dL) & $15.7 \pm 0.3$ & $16.3 \pm 0.5$ & $16.1 \pm 0.3$ & $16.8 \pm 0.9^{*}$ & $16.8 \pm 0.8$ & $16.6 \pm 0.7$ & $16.2 \pm 0.7$ & $15.8 \pm 0.3$ \\
\hline HCT $(\%)$ & $52.0 \pm 1.4$ & $54.5 \pm 1.1$ & $53.2 \pm 0.8$ & $55.8 \pm 3.1^{*}$ & $53.9 \pm 3.1$ & $53.5 \pm 2.2$ & $52.4 \pm 1.5$ & $51.3 \pm 0.9$ \\
\hline MCV (fL) & $58.7 \pm 1.0$ & $58.1 \pm 1.0$ & $58.3 \pm 2.1$ & $58.5 \pm 2.2$ & $60.3 \pm 1.9$ & $60.0 \pm 1.3$ & $61.0 \pm 1.0$ & $59.2 \pm 1.5$ \\
\hline $\mathrm{MCH}(\mathrm{pg})$ & $17.7 \pm 0.3$ & $17.4 \pm 0.3$ & $17.6 \pm 0.6$ & $17.6 \pm 0.7$ & $18.8 \pm 0.5$ & $18.6 \pm 0.5$ & $18.9 \pm 0.2$ & $18.2 \pm 0.4$ \\
\hline $\mathrm{MCHC}(\mathrm{g} / \mathrm{dL})$ & $30.2 \pm 0.4$ & $29.9 \pm 0.4$ & $30.3 \pm 0.5$ & $30.1 \pm 0.3$ & $31.2 \pm 0.2$ & $31.0 \pm 0.6$ & $31.0 \pm 0.4$ & $30.7 \pm 0.4$ \\
\hline $\operatorname{PLT}\left(10^{3} / \mu \mathrm{L}\right)$ & $927 \pm 206$ & $959 \pm 188$ & $920 \pm 92$ & $1047 \pm 149$ & $1169 \pm 174$ & $1197 \pm 162$ & $1129 \pm 157$ & $1076 \pm 52$ \\
\hline $\operatorname{WBC}\left(10^{3} / \mu \mathrm{L}\right)$ & $8.55 \pm 1.52$ & $9.89 \pm 2.36$ & $9.09 \pm 1.26$ & $10.9 \pm 3.54$ & $5.88 \pm 0.71$ & $6.06 \pm 1.59$ & $6.46 \pm 1.22$ & $6.19 \pm 2.15$ \\
\hline NEU $(\%)$ & $18.3 \pm 7.4$ & $24.2 \pm 9.4$ & $18.3 \pm 5.2$ & $23.6 \pm 8.1$ & $15.2 \pm 5.3$ & $16.8 \pm 4.8$ & $13.6 \pm 0.4$ & $15.6 \pm 6.3$ \\
\hline $\operatorname{Lym}(\%)$ & $78.6 \pm 7.2$ & $72.5 \pm 9.2$ & $77.8 \pm 6.3$ & $72.1 \pm 8.5$ & $80.4 \pm 5.6$ & $78.2 \pm 4.7$ & $82.0 \pm 2.1$ & $80.6 \pm 6.1$ \\
\hline MONO (\%) & $1.14 \pm 0.36$ & $1.38 \pm 0.34$ & $1.48 \pm 0.28$ & $2.24 \pm 0.36^{*}$ & $1.40 \pm 0.43$ & $1.26 \pm 0.30$ & $1.46 \pm 0.25$ & $1.32 \pm 0.15$ \\
\hline EOS (\%) & $1.26 \pm 0.57$ & $1.30 \pm 0.17$ & $1.88 \pm 1.11$ & $1.40 \pm 0.25$ & $2.22 \pm 0.41$ & $3.14 \pm 2.08$ & $2.48 \pm 1.60$ & $1.94 \pm 0.61$ \\
\hline Baso (\%) & $0.70 \pm 0.07$ & $0.56 \pm 0.15$ & $0.56 \pm 0.25$ & $0.68 \pm 0.15$ & $0.78 \pm 0.23$ & $0.56 \pm 0.18$ & $0.46 \pm 0.27$ & $0.52 \pm 0.19$ \\
\hline
\end{tabular}

RBC, erythrocyte count; $\mathrm{HGB}$, hemoglobin; $\mathrm{HCT}$, hematocrit; $\mathrm{MCV}$, mean corpuscular volume; $\mathrm{MCH}$, mean corpuscular hemoglobin; MCHC, mean corpuscular hemoglobin concentration; PLT, platelet count; WBC, total leucocyte counts; NEU, neutrophils; Lym, lymphocytes; MONO, monocytes; EOS, eosinophils; and Baso, basophils.

All data are given as mean \pm standard deviation.

*Significantly different from vehicle control at $p<0.05$. 
Table 3. Serum biochemical values of male and female rats in the 90-day repeated oral dose toxicity study of ARAE

\begin{tabular}{|c|c|c|c|c|c|c|c|c|}
\hline \multirow{3}{*}{ Parameter } & \multicolumn{8}{|c|}{ Dose (mg/kg/day) } \\
\hline & \multicolumn{4}{|c|}{ Male } & \multicolumn{4}{|c|}{ Female } \\
\hline & 0 & 500 & 1,000 & 2,000 & 0 & 500 & 1,000 & 2,000 \\
\hline AST (IU/L) & $89.4 \pm 23.0$ & $99.6 \pm 31.5$ & $95.4 \pm 21.3$ & $117 \pm 41.9$ & $81.4 \pm 12.1$ & $86.2 \pm 14.4$ & $83.8 \pm 18.0$ & $86.4 \pm 15.7$ \\
\hline ALT (IU/L) & $49.4 \pm 16.8$ & $45.8 \pm 7.9$ & $44.2 \pm 8.3$ & $60.8 \pm 29.4$ & $31.2 \pm 5.4$ & $33.6 \pm 3.9$ & $31.6 \pm 3.1$ & $31.0 \pm 6.7$ \\
\hline ALP (IU/L) & $307 \pm 44$ & $299 \pm 43$ & $282 \pm 38$ & $305 \pm 47$ & $179 \pm 31$ & $201 \pm 47$ & $171 \pm 19$ & $164 \pm 24$ \\
\hline CPK (IU/L) & $225 \pm 122$ & $182 \pm 61$ & $245 \pm 72$ & $231 \pm 88$ & $301 \pm 166$ & $283 \pm 147$ & $311 \pm 217$ & $314 \pm 248$ \\
\hline GGT (IU/L) & $1.20 \pm 1.30$ & $1.00 \pm 0.71$ & $1.00 \pm 0.00$ & $1.25 \pm 1.50$ & $0.50 \pm 0.58$ & $2.25 \pm 1.71$ & $0.40 \pm 0.55$ & $1.00 \pm 1.73$ \\
\hline BUN (mg/dL) & $18.2 \pm 4.1$ & $17.0 \pm 2.2$ & $19.0 \pm 2.0$ & $21.0 \pm 3.4$ & $17.0 \pm 1.9$ & $20.8 \pm 2.2$ & $20.0 \pm 3.4$ & $19.0 \pm 2.5$ \\
\hline GLU (mg/dL) & $218 \pm 21$ & $231 \pm 65$ & $249 \pm 39$ & $275 \pm 42$ & $142 \pm 26$ & $165 \pm 32$ & $168 \pm 38$ & $156 \pm 52$ \\
\hline ALB (g/dL) & $2.78 \pm 0.15$ & $2.70 \pm 0.12$ & $3.00 \pm 0.22$ & $3.08 \pm 0.16^{*}$ & $3.02 \pm 0.30$ & $3.22 \pm 0.11$ & $3.24 \pm 0.15$ & $2.96 \pm 0.30$ \\
\hline $\mathrm{TP}(\mathrm{g} / \mathrm{dL})$ & $7.50 \pm 0.19$ & $7.38 \pm 0.18$ & $8.10 \pm 0.57$ & $8.24 \pm 0.48^{*}$ & $7.54 \pm 0.54$ & $7.72 \pm 0.13$ & $7.60 \pm 0.31$ & $7.24 \pm 0.59$ \\
\hline $\mathrm{A} / \mathrm{G}$ (ratio) & $0.63 \pm 0.02$ & $0.63 \pm 0.01$ & $0.63 \pm 0.01$ & $0.63 \pm 0.01$ & $0.60 \pm 0.01$ & $0.58 \pm 0.01$ & $0.57 \pm 0.01$ & $0.59 \pm 0.02$ \\
\hline T-CHO (mg/dL) & $78.0 \pm 18.0$ & $69.4 \pm 13.5$ & $74.8 \pm 10.5$ & $77.2 \pm 5.0$ & $98.4 \pm 10.9$ & $93.4 \pm 15.5$ & $90.6 \pm 8.4$ & $95.8 \pm 31.7$ \\
\hline $\mathrm{CRE}(\mathrm{mg} / \mathrm{dL})$ & $0.60 \pm 0.10$ & $0.62 \pm 0.04$ & $0.70 \pm 0.07$ & $0.66 \pm 0.05$ & $0.62 \pm 0.04$ & $0.68 \pm 0.08$ & $0.68 \pm 0.08$ & $0.68 \pm 0.08$ \\
\hline TG (mg/dL) & $77.8 \pm 21.8$ & $124 \pm 25.1$ & $111 \pm 54.6$ & $104 \pm 23.1$ & $47.6 \pm 11.3$ & $46.0 \pm 21.4$ & $63.8 \pm 28.9$ & $70.0 \pm 26.5$ \\
\hline IP (mg/dL) & $10.0 \pm 0.7$ & $10.4 \pm 0.4$ & $10.6 \pm 0.7$ & $11.3 \pm 1.2$ & $10.0 \pm 0.6$ & $10.4 \pm 0.9$ & $10.1 \pm 1.3$ & $10.5 \pm 0.6$ \\
\hline $\mathrm{Ca}(\mathrm{mg} / \mathrm{dL})$ & $11.9 \pm 0.6$ & $12.0 \pm 0.6$ & $13.0 \pm 1.2$ & $13.6 \pm 0.6$ & $11.6 \pm 0.7$ & $11.6 \pm 0.3$ & $11.8 \pm 0.2$ & $11.6 \pm 0.4$ \\
\hline
\end{tabular}

AST, aspartate aminotransferase; ALT, alanine aminotransferase; ALP, alkaline phosphatase; CPK, creatine phosphokinase; GGT, gamma glutamyltranspeptidase; BUN, blood urea nitrogen; GLU, glucose; ALB, albumin; TP, total protein; A/G, albumin/globulin; T-CHO, total cholesterol; CRE, creatinine; TG, triglycerides; IP, inorganic phosphorus; and Ca, calcium.

All data are given as mean \pm standard deviation.

*Significantly different from vehicle control at $p<0.05$.

124.4 and $138.9\left(\times 10^{6} / \mathrm{g}\right)$, and the incidence of sperm abnormalities in the epididymis was $2.0,3.4,2.1$ and $2.1 \%$, respectively. There were no significant effects on any sperm parameter (data not shown).

Organ weights. Absolute spleen weight in males of the $500 \mathrm{mg} / \mathrm{kg} /$ day group increased significantly when compared with that in the vehicle control group $(p<0.05)$. However, this difference was not considered treatmentrelated since it was very slight without a dose-response relationship and was absent in females of the treatment groups. The organ weight data were not shown for the recovery groups because there were no important findings at terminal sacrifice.

Histopathology. Minimal hepatocyte vacuolation was observed in one female in the $2,000 \mathrm{mg} / \mathrm{kg} / \mathrm{day}$ group, and focal myocarditis was observed in two, one and two males in the vehicle control, 500 and $2,000 \mathrm{mg} / \mathrm{kg} /$ day groups, respectively. Chronic progressive nephropathy in the kid-

Table 4. Histopathological findings of male and female rats in the 90-day repeated oral dose toxicity study of ARAE

\begin{tabular}{|c|c|c|c|c|c|c|c|c|}
\hline \multirow{3}{*}{$\begin{array}{c}\text { Parameters } \\
\text { (organ/findings) }\end{array}$} & \multicolumn{8}{|c|}{ Dose (mg/kg/day) } \\
\hline & \multicolumn{4}{|c|}{ Male } & \multicolumn{4}{|c|}{ Female } \\
\hline & 0 & 500 & 1,000 & 2,000 & 0 & 500 & 1,000 & 2,000 \\
\hline \multicolumn{9}{|l|}{ Liver } \\
\hline No specific lesion & 5 & 5 & 5 & 5 & 5 & 5 & 5 & 4 \\
\hline Vacuolation, hepatocyte & 0 & 0 & 0 & 0 & 0 & 0 & 0 & 1 \\
\hline \multicolumn{9}{|l|}{ Heart } \\
\hline No specific lesion & 3 & 4 & 5 & 3 & 5 & 5 & 5 & 5 \\
\hline Myocarditis, focal & 2 & 1 & 0 & 2 & 0 & 0 & 0 & 0 \\
\hline \multicolumn{9}{|l|}{ Kidneys } \\
\hline No specific lesion & 3 & 4 & 3 & 4 & 4 & 5 & 4 & 4 \\
\hline Basophilic tubules, focal & 0 & 0 & 1 & 0 & 0 & 0 & 1 & 0 \\
\hline Cell infiltration, lymphocytic, focal, interstitium & 1 & 0 & 0 & 1 & 0 & 0 & 0 & 0 \\
\hline Cyst & 0 & 0 & 0 & 0 & 0 & 0 & 0 & 1 \\
\hline Hyaline cast & 1 & 1 & 2 & 0 & 1 & 0 & 0 & 0 \\
\hline
\end{tabular}

The other organs examined were excluded from this table, since no histopathological alterations were observed. 
ney was observed in two, one, two and one males in the vehicle control, 500, 1,000 and 2,000 mg/ $\mathrm{kg} /$ day groups, and in one, one and one females in the vehicle control, 1,000 and $2,000 \mathrm{mg} / \mathrm{kg} /$ day groups, respectively (Table 4). At the end of recovery period, focal mononuclear cell infiltration in the liver and focal myocarditis were observed in one female each in the vehicle control group. Chronic progressive nephropathy was observed in two males in the $2,000 \mathrm{mg} / \mathrm{kg} /$ day group and in one female in the vehicle control group, but there were no obvious differences in the incidence and severity of the finding between the groups. No other macroscopic or microscopic lesions were observed.

\section{DISCUSSION}

$\mathrm{AR}$ is known to have diuretic and damp-heat clearing actions and has been used for the treatment of dysuria, edema and urinary tract infections in traditional medicine (20). Despite its long history of usage in treating a wide range of ailments, the potential adverse effects of AR are not fully characterized. The present study aimed to evaluate the potential toxicity of the ARAE following 90-day repeated oral administration to male and female rats at doses of 0 (control), 500, 1,000 and 2,000 mg/kg body weight, and determine the gross toxicity, NOAEL, and target organs. Additional recovery groups from the control and high dose groups were observed for a 28-day recovery period.

Chromatograms of ARAE detected main compounds with four peaks: compound $1\left(\mathrm{~m} / \mathrm{z} 281.1641[\mathrm{M}+\mathrm{H}]^{+}\right)$, compound $2\left(\mathrm{~m} / \mathrm{z} 295.1812[\mathrm{M}+\mathrm{H}]^{+}\right)$, compound $3(\mathrm{~m} / \mathrm{z}$ $\left.265.1696[\mathrm{M}+\mathrm{H}]^{+}\right)$and compound $4\left(\mathrm{~m} / \mathrm{z} 279.1854[\mathrm{M}+\mathrm{H}]^{+}\right)$. These compound 1 ((R)-1-(1-phenyl-3,4-dihydropyrrolo[1,2a]pyrazin-2(1H)-yl)pent-4-en-1-one (101c); $\mathrm{C}_{18} \mathrm{H}_{21} \mathrm{~N}_{2} \mathrm{O}$ ), compound 2 (trans-N-Benzyl-1-methyl-4-phenylpyrrolidine-3-carboxamide; $\mathrm{C}_{19} \mathrm{H}_{23} \mathrm{~N}_{2} \mathrm{O}$ ), compound $3(\mathrm{~N}-\{(\mathrm{E}, 2 \mathrm{E})$ 2-[(2,6-Dimethylphenyl)imino] ethyliden \}-2,6-dimethylaniline; $\mathrm{C}_{18} \mathrm{H}_{21} \mathrm{~N}_{2}$ ) and compound 4 ((E)-N-(2-phenyl-1(piperidin-1-yl)ethylidene)aniline; $\mathrm{C}_{19} \mathrm{H}_{23} \mathrm{~N}_{2}$ ) were identified as phenolic compounds based on MS data and reference (12,21-24).

According to the previous reports, alisol A 24-acetate $\left(\mathrm{m} / \mathrm{z} 531[\mathrm{M}-\mathrm{H}]^{-}\right)$is a main diuretic compound of alcoholic AR extract $(14,25)$. Alisol B 23-acetate $(\mathrm{m} / \mathrm{z} 514$ $\left[\mathrm{M}^{+}, \mathrm{C}_{32} \mathrm{H}_{50} \mathrm{O}_{5}\right)$ and alisol C 23-acetate $\left(\mathrm{m} / \mathrm{z} 528\left[\mathrm{M}^{+}\right.\right.$, $\mathrm{C}_{32} \mathrm{H}_{48} \mathrm{O}_{6}$ ) were identified as the major constituents isolated from AR ethanol extract (26). These results of the previous studies and ours showed major constituent differences between aqueous and ethanol extracts of AR. However, the definite structures need further structural analysis and elucidation.

Under the conditions of our study, 90-day repeated oral administration of the ARAE did not result in clinical signs of toxicity or death at any dose tested in both genders. No abnormal changes in body weight and food consumption were observed during the treatment or recovery period.

The significant changes in hematological and serum biochemical parameters observed in the treatment groups including an increase in the RBC, HGB, HCT, ALB, and TP in males of the $2,000 \mathrm{mg} / \mathrm{kg} /$ day group were considered as treatment-related effects. In addition, the significant increase in urine volume observed in males of the $2,000 \mathrm{mg} / \mathrm{kg} /$ day group was also considered as a treatment-related effect. Preliminary pharmacological investigations have shown that the alisol A 24-monoacetate content in AR has a similar diuretic effect with hydrochlorothiazide and is an active component that is responsible for the diuretic activity of AR (14). These hematological and serum biochemical changes occur by diuretic or dehydration related loss of fluids (27-29). However, the increased hematological and serum biochemical changes were not considered toxicologically significant because they were within the limits of normal biological variation (30-32) and unaccompanied by systemic abnormalities and correlative histopathological findings. In addition, diuretic or dehydration toxicity signs such as weight loss, changes of blood urea nitrogen, creatinine and histopathology of the urinary tract were not observed at any dose tested. The absence of treatment-related effects in hematological and serum biochemical parameters in female rats, further supports the view that these effects are not toxicologically important. On the other hand, a previous chronic toxicity research of AR ethanol extract showed a few remarkable changes in liver weight and serum levels of ALT, AST and total bilirubin in female rats (17). The results of the previous report (17) and our study have also shown effect differences between ethanol and aqueous extracts of AR.

No abnormal changes in estrous cycle, sperm parameters and necropsy findings were observed at any dose tested. The significant increase of absolute spleen weight observed in males of the $500 \mathrm{mg} / \mathrm{kg} /$ day group was not considered treatment-related due to lack of supportive serum biochemical and histopathological findings, and was within the limits of normal reference data (31).

The principal histopathological finding observed in the vehicle control group and treatment groups was chronic progressive nephropathy in the kidney. However, the lesion was considered as background pathology unrelated to the test substance, because the frequency and severity of observation were similar to the results in the vehicle control group. Chronic progressive nephropathy is a specific disease commonly observed in elderly male rats and a lack of relevance has been reported on the extrapolation to human (33-35).

According to the previous reports, a single oral administration of ARAE did not induce any toxic effect at a dose of $\leq 4,000 \mathrm{mg} / \mathrm{kg}$ in rats (18), and the NOAEL in a 14-day 
repeated oral dose toxicity study was assumed as over $2,000 \mathrm{mg} / \mathrm{kg} /$ day (19). A 60 -day oral dose toxicity study showed that some renal function parameters can be affected by a high dose $(33.3 \mathrm{~g} / \mathrm{kg} /$ day $)$ of ARAE in male rats, but histopathological changes were not observed at any doses tested (15). O-Ryeing-San (combination of crude components of ARAE 33.5\%) showed no genotoxicity in the Ames test, chromosome aberration test, and micronucleus test (36). The results of the present study also showed that a 90-day repeated oral administration of the ARAE did not cause any significant adverse effect at a dose level of $\leq 2,000 \mathrm{mg} / \mathrm{kg} /$ day in rats. The results of earlier studies and ours clearly showed that repeated oral administration of ARAE to rats is safe up to $2,000 \mathrm{mg} / \mathrm{kg}$ / day.

In conclusion, the results of the present study showed that 90-day repeated oral administration of the ARAE produced no significant toxic effects in rats. Under the present experimental conditions, the no-observed-adverse-effect level (NOAEL) was $>2,000 \mathrm{mg} / \mathrm{kg} /$ day for both genders, and no target organs were identified.

\section{ACKNOWLEDGMENTS}

This study was part of a project (Safety of the Korean Herbal Medicine, N17001) funded by the Division of Tradition Korean Medicine Research of the National Development Institute of Korean Medicine. This research was also supported by Basic Science Research Program through the National Research Foundation of Korea (NRF) funded by the Ministry of Education [NRF-2016R1D1A2B04936124].

\section{CONFLICT OF INTEREST}

The authors declare that there is no conflict of interest.

Received August 22, 2018; Accepted November 6, 2018

\section{REFERENCES}

1. Choi, Y.J., Yoon, Y.J., Choi, H.S., Park, S.R., Oh, S.H., Jeong, S.M., Suh, H.R. and Lee B.H. (2011) Effects of medicinal herb extracts and their components on steatogenic hepatotoxicity in Sk-hep1 cells. Toxicol. Res., 27, 122-216.

2. Lee, J.C., Lee, E. and Lee, Y.C. (2008) Effects of Rhizoma Alismatis on lipid composition and TBARS concentration in rat fed high fat diet. Kor. J. Herbol., 23, 113-117.

3. Kim, S.E., Rhyu, D.Y., Yokozawa, T. and Park, J.C. (2007) Antioxidant effect of Alisma plantago-aquaticavar. orientale and its main component. Kor. J. Pharmacogn., 38, 372375.

4. National Institute of Medicinal Materials (2004) Medicinal Plants and Animals, Science and Technology. National Institute of Medicinal Materials, Hanoi.

5. Tian, T., Chen, H. and Zhao, Y.Y. (2014) Traditional uses, phytochemistry, pharmacology, toxicology and quality control of Alisma orientale (Sam.) Juzep: a review. J. Ethnopharmacol., 158, 373-387.

6. Lee, S.I. (1981) Studies on the diuretic action of Oryeongsan and Kami-Oryengsan. Kor. J. Pharmacogn, 12, 31-43.

7. Ahn, M.J., Lee, C.H., Shin,Y.W., Chun, M.S., Kim, C.Y. and Kim, J. (2008) Determination of alisol B 23-acetate and alisol C 23-acetate in Alismatis rhizoma by HPLC-ESI-MS. Nat. Prod. Sci., 14, 152-155.

8. Huang, D.S. and Shin, H.K. (2009) A study on compositions and dosages of Yukmijihwang-Hwan by literature review on the classics of oriental medicine. Kor. J. Orient. Med. Prescr., 17, 17-28.

9. Yu, D.J., Yoon, K.M., Jang, S.Y., Lee, Y.K. and Kang, S.B. (2009) Nephroprotective effects of Taeksa-San aqueous extracts on cisplatin-induced rat acute renal failure. Kor. $J$. Orient. Int. Med., 30, 832-844.

10. Mi, Q.W., Cao, Z.G., Liu, J.H., Wu, J.Z., Yin, C.P., Zhou, S.W. and Ye, Z.Q. (2005) Effects of active fraction of Alisma orientale on osteopontin expression in renal tissue of urolithiasis model rat with calcium oxalate stone. Chin. Tradit. Herbal Drugs, 36, 1827-1830.

11. Jiang, Z.Y., Zhang, X.M., Zhang, F.X., Liu, N., Zhao, F., Zhou, J. and Chen, J.J. (2006) A new triterpene and antihepatitis B virus active compounds from Alisma orientalis. Planta Med., 72, 951-954.

12. Zhao, M., Chen, J.Y., Xu, L.J., Goedecke, T., Zhang, X.Q., Duan, J.A. and Che, C.T. (2012) Cis-aconitic anhydride ethyl ester and phenolic compounds from the seeds of Alisma orientale. Nat. Prod. Commun., 7, 785-787.

13. Tomoda, M., Gonda, R., Shimizu, N. and Ohara, N. (1994) Characterization of an acidic polysaccharide having immunological activities from the tuber of Alisma orientale. Biol. Pharm. Bull., 17, 572-576.

14. Wang, L.X.,Wu, Q.N., Zhang, Q., Peng, G.P. and Ding, A.W. (2008) Basic study of diuretic active compounds in Rhizoma Alismatis. West China J. Pharm. Sci., 23, 670-672.

15. Duan, X.Y., Wang, J.B., Yin, X.L., Yan, S.F. and Xiao, Y. (2004) A 60-day feeding study of Rhizoma Alismatis Orientalis in SD rats. Chin. J. Food Hyg., 16, 108-111.

16. Zhu, J.H., Bao, X.R., He, H.P. and Zhang, Q. (2007) Experimental studies of nephrotoxicity induced by Alisma orientalis in rats. Pharmacol. Clin. Chin. Mater. Med., 23, 60-62.

17. Huang, M.Q., Xu, W., Wu, S.S., Lu, J.J. and Chen, X.P. (2013) A 90-day subchronic oral toxicity study of triterpeneenriched extract from Alismatis rhizoma in rats. Food Chem. Toxicol., 58, 318-323.

18. Seok, J.H., Roh, H.S., Jeong, J.Y. and Ha, H.Y. (2013) Acute oral toxicity of Alismatis rhizoma in SD rats. J. Kor. Med. Ophthalmol. Otolaryngol. Dermatol., 26, 15-25.

19. Roh, H.S., Seok, J.H., Jeong, J.Y., Lee, J.K., Kim, T.S., Choi, H.K. and Ha, H.Y. (2014) Repeated dose oral toxicity study of Alismatis rhizoma in SD rats. J. Kor. Med. Ophthalmol. Otolaryngol. Dermatol., 27, 79-90.

20. Chang, H.M. and But, P.P.H. (1987) Pharmacology and Applications of Chinese Materia Medica (vol. II), World Scientific Publishing Co., Singapore, pp. 802-805.

21. Kreituss, I. (2016) Kinetic resolution of cyclic secondary amines and synthesis of partially saturated N-heterocycles. 
Swiss Federal Institute of Technology (ETH Zurich), ETH 23440.

22. Buyev, E.M., Moshkin, V.S. and Sosnovsky, V.Ya. (2017) $(3+2)$ Cycloaddition of N-methylazomethinylide, obtained from sarcosine and formaldehyde, to enones and enamides with $\mathrm{CH}-$ and NH-acidity. Chem. Heterocycl. Compd., 53, 167-172.

23. Müller, C. (2006) Studies on Olefin Metathesis and Its Application in the Synthesis of Middle Rings. Department of Chemistry, University of Dortmund.

24. Dai, Q., Jiang, Y., Yu, J.T. and Cheng, J. (2015) Palladiumcatalyzed three-component reaction of N-tosyl hydrazones, isonitriles and amines leading to amidines. Chem. Commun., 51, 16645.

25. Makabel, B., Zhao, Y., Wang, B., Bai, Y., Zhang, Q., Wu, L. and Lv, Y. (2008) Stability and structure studies on alisol A 24-acetate. Chem. Pharm. Bull., 56, 41-45.

26. Lee, J.H., Kwon, O.S., Jin, H.G., Woo, E.R., Kim, Y.S. and Kim, H.P. (2012) The Rhizomes of Alisma orientale and alisol derivatives inhibit allergic response and experimental atopic dermatitis. Biol. Pharm. Bull., 35, 1581-1587.

27. Morris, M. (1982) Neurohypophyseal response to dehydration in the spontaneously hypertensive rat. Hypertension, $\mathbf{4}$, 161-166.

28. Ogawa, Y., Aoki, K., Kato, J. and Iwasaki, K.I. (2013) Differential effects of mild central hypovolemia with furosemide administration vs. lower body suction on dynamic cerebral autoregulation. J. Appl. Physiol., 114, 211-216.

29. Feng, Y.L., Chen, H., Tian, T., Chen, D.Q., Zhao, Y.Y. and Lin, R.C. (2014) Diuretic and antidiuretic activities of the ethanol and aqueous extracts of Alismatis rhizoma. J. Ethno- pharmacol., 154, 386-390.

30. Petterino, C. and Argentino-Storino, A. (2006) Clinical chemistry and haematology historical data in control Sprague-Dawley rats from pre-clinical toxicity studies. Exp. Toxicol. Pathol., 57, 213-219.

31. Han, Z.Z., Xu, H.D., Kim, K.H., Ahn, T.H., Bae, J.S., Lee, J.Y., Gil, K.H., Lee, J.Y., Woo, S.J., Yoo, H.J., Lee, H.K., Kim, K.H., Park, C.K., Zhang, H.S. and Song, S.W. (2010) Reference data of the main physiological parameters in control Sprague-Dawley rats from pre-clinical toxicity studies. Lab. Anim. Res., 26, 153-164.

32. Lee, J.M., Lee, M.A., Do, H.N., Song, Y.I., Bae, R.J.N., Lee, H.Y., Park, S.H., Kang, J.S. and Kang, J.K. (2012) Historical control data from 13-week repeated toxicity studies in Crj: CD (SD) rats. Lab. Anim. Res., 28, 115-121.

33. Hard, G.C. and Khan, K.N. (2004) A contemporary overview of chronic progressive nephropathy in the laboratory rat, and its significance for human risk assessment. Toxicol. Pathol., 32, 171-180.

34. Wolf, D.C. and Mann, P.C. (2005) Confounders in interpreting pathology for safety and risk assessment. Toxicol. Appl. Pharmacol., 202, 302-308.

35. Hard, G.C., Johnson, K.J. and Cohen, S.M. (2009) A comparison of rat chronic progressive nephropathy with human renal disease-implications for human risk assessment. Crit. Rev. Toxicol., 39, 332-346.

36. Lee, M.Y., Seo, C.S., Kim, J.Y. and Shin, H.K. (2015) Genotoxicity evaluation of Oryeong-san water extract using in vitro and in vivo tests. BMC Complement. Altern. Med., 15, 273. 